\title{
A!
}

This is an electronic reprint of the original article.

This reprint may differ from the original in pagination and typographic detail.

Wang, X.; Asadchy, V. S.; Tretyakov, S. A.

\section{Multiple-Beam Power Combining Using Space-Time Metasurfaces}

Published in:

2021 15th International Congress on Artificial Materials for Novel Wave Phenomena, Metamaterials 2021

DOI:

10.1109/Metamaterials52332.2021.9577186

Published: 20/09/2021

Document Version

Peer reviewed version

Please cite the original version:

Wang, X., Asadchy, V. S., \& Tretyakov, S. A. (2021). Multiple-Beam Power Combining Using Space-Time Metasurfaces. In 2021 15th International Congress on Artificial Materials for Novel Wave Phenomena,

Metamaterials 2021 (pp. 460-462). IEEE. https://doi.org/10.1109/Metamaterials52332.2021.9577186

This material is protected by copyright and other intellectual property rights, and duplication or sale of all or part of any of the repository collections is not permitted, except that material may be duplicated by you for your research use or educational purposes in electronic or print form. You must obtain permission for any other use. Electronic or print copies may not be offered, whether for sale or otherwise to anyone who is not an authorised user. 


\title{
Multiple-Beam Power Combining Using Space-Time Metasurfaces
}

\author{
X. Wang ${ }^{1}$, V. S. Asadchy ${ }^{2}$, and S. A. Tretyakov ${ }^{1}$ \\ ${ }^{1}$ Department of Electronics and Nanoengineering, School of Electrical Engineering, Aalto University, \\ P.O. Box 15500, FI-00076 Aalto, Finland \\ ${ }^{2}$ Ginzton Laboratory and Department of Electrical Engineering, Stanford University, Stanford, CA 94305, \\ USA \\ xuchen.wang@aalto.fi
}

\begin{abstract}
In this talk, we will present a new concept of space-time power combiners which allow perfect power concentration of plane waves from multiple input directions to a single wave propagating into the desired output direction. Such functionality can be realized in metasurfaces with travelling-wave modulations of the surface impedance. The spatiotemporal modulation imparts different spatial and temporal momenta on the incident beams, such that their reflected beams are spatially collimated and their powers are incoherently summed. The proposed technique has very important applications in unidirectional guiding of electromagnetic signals among multiple routes, as well as in high-power laser technologies.
\end{abstract}

\section{INTRODUCTION}

Power combination from multiple input ports to a single output port is an inverse function of beam splitting. In principle, a beam splitter can serve as a power combiner if the input and output ports are interchanged. However, such power combiners have significant drawbacks, because the input signals must be coherent with balanced amplitudes and phases before entering into the combiner [1,2]. This drawback has significantly prevented the wide applications of microwave power combiners. In principle, in a passive and linear system, it is not possible to combine several waves with random amplitudes and phases to a single port without losing some part of incident energy [2].

In optics, efficient power combination is highly demanded in many areas, such as high-power lasers. Due to the strict requirements for beam amplitudes and phases in coherent combiners, many early works focus on the combination of incoherent beams $[3,4]$. The principle is simple: The sources at different spatial positions emit beams at different frequencies, $\omega_{1}, \omega_{2}, \cdots$, and $\omega_{n}$. These incoherent beams are shed onto spatially dispersive structures (e.g., diffraction gratings), such that they can be combined in a single direction without coherent interference. However, these devices need accurate incoherence (frequency difference) among different emitters and strict mechanical stability of the combing setup. In addition, the method cannot be generalized to microwave and millimeter waves for sources having small frequency differences $\Delta f$, because the size of the diffraction gratings scales as $1 / \Delta f[3,5]$.

Recent advances in time-modulated materials have attracted significant attention of microwave and optics communities. Dynamically varying materials bring additional engineering freedoms and thus can break the theoretical and technical restrictions imposed on traditional time-invariant systems [6-12]. In this presentation, we will show that by modulating a metasurface in space and time in a traveling-wave fashion, free-space waves coming from different directions can be collimated into a single direction, without losses of incident energy [see Fig. 1]. The device is based on the following principle: Spatial modulation imparts spatial momenta on the incident beams such that they can be directed into the same desired direction, while temporal modulation imparts different temporal momenta (frequencies) so that the output powers of the incident beams are incoherently summed. Thus, the reflected waves at the output port will have generally different frequencies separated from one another by a multiple of the modulation frequency $\omega_{\mathrm{m}}$. Nevertheless, for a receiver with given bandwidth it is always possible to choose sufficiently small $\omega_{\mathrm{m}}$ to ensure that all these frequency harmonics are received. 


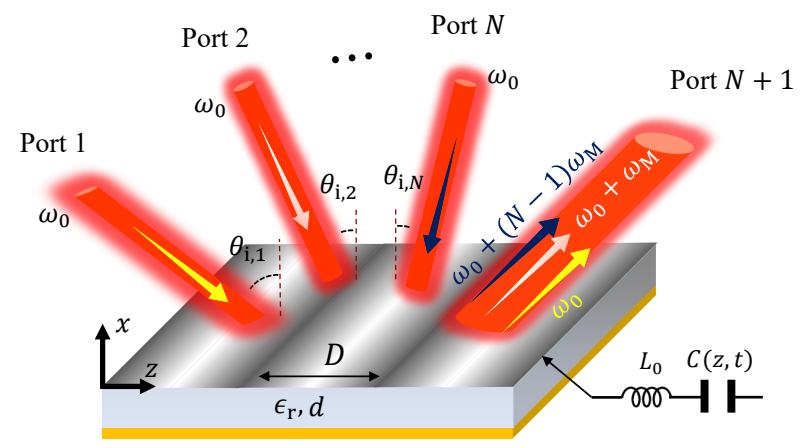

(a)

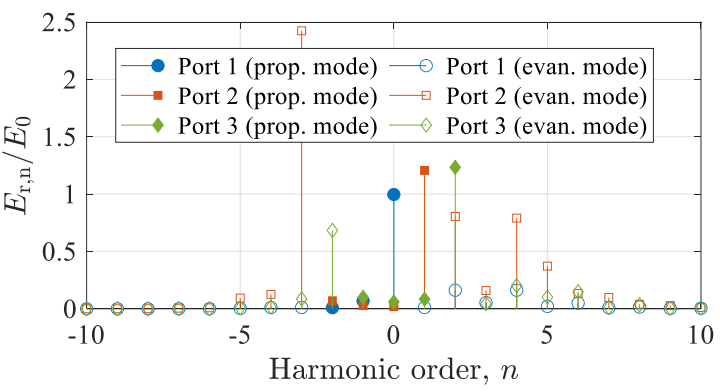

(b)

Fig. 1: (a) Schematics of a general $N$-port power combiner. (b) Scattering harmonics of a three-port combiner for incidences from Ports 1, 2, and 3. The evanescent and propagating harmonics are distinguished in the figure. The design parameters are fixed as $\epsilon_{\mathrm{r}}=4, d=9.8 \mathrm{~mm}, \omega_{0}=2 \pi \times 10 \mathrm{GHz}, C(z, t)=13\left[1+0.0265 \cos \left(\beta_{\mathrm{M}} z-\right.\right.$ $\left.\left.\omega_{\mathrm{M}} t\right)-0.3837 \cos \left(2 \beta_{\mathrm{M}} z-2 \omega_{\mathrm{M}} t+0.06 \pi\right)\right]$ with the unit of $\mathrm{fF}$, and $L_{0}=18.5 \mathrm{nH}$.

\section{BASIC CONCEPT}

Let us consider a metasurface composed of a spatiotemporally varying sheet (modelled by its sheet impedance) on a grounded substrate, as shown in Fig. 1(a). The sheet impedance is represented by a series connection of capacitance $C(z, t)$ and inductance $L_{0}$. The capacitance is modulated with a general traveling waveform

$$
C(z, t)=\sum_{m=-\infty}^{+\infty} c_{m} e^{-j m\left(\beta_{\mathrm{M}} z-\omega_{\mathrm{M}} t\right)}
$$

where $\omega_{\mathrm{M}}$ is the modulation frequency, $c_{m}^{*}=c_{-m}$ are the Fourier terms of the modulation function, and $\beta_{\mathrm{M}}=$ $2 \pi / D$ is the modulation wavenumber ( $D$ is the spatial periodicity of the metasurface). According to the Floquet theory, a wave incident from $\theta=\theta_{\mathrm{i}}$ is scattered into multiple spatial modes, and the $n$-th mode propagates with the tangential wavevector $k_{z n}=k_{0} \sin \theta_{\mathrm{i}}+n \beta_{\mathrm{M}}$ and at the frequency of $\omega_{n}=\omega_{0}+n \omega_{\mathrm{M}}$. By properly choosing the spatial $\beta_{\mathrm{M}}$ and temporal $\omega_{\mathrm{M}}$ modulation frequencies, waves incident from different directions can share a common scattering channel. Figure 1(a) shows a general $N$-port space-time system, where waves from Port 1 to Port $N$ are forced to combine at Port $N+1$.

Let us consider a TE-polarized plane wave incident from Port 1 at the angle $\theta_{\mathrm{i}, 1}$ and define its specular direction as the common receiving port. Then, we require that waves from Port $n(1 \leq n \leq N)$ are scattered to the same output Port $N+1$, corresponding to their $n-1$ mode. Therefore, for a wave incident from Port $n$, the following relation must hold:

$$
k_{0} \sin \theta_{\mathrm{i}, n}+\frac{(n-1) 2 \pi}{D}=\alpha_{n} k_{0} \sin \theta_{\mathrm{i}, 1},
$$

where $\alpha_{n}=\left|\frac{\omega_{0}+(n-1) \omega_{\mathrm{M}}}{\omega_{0}}\right|$. If $D, \omega_{\mathrm{M}}$, and $\theta_{\mathrm{i}, 1}$ are specified, the angles of Ports $n$, i.e., $\theta_{\mathrm{i}, n}$, can be uniquely determined by Eq. (2). As soon as the directions of all ports are determined, we can engineer the modulation waveform, i.e., $c_{m}$ in Eq. (1), so that the incident power from either input port is perfectly channeled to Port $N+1$ (independently how many input channels are excited at the same time). In the reference [13], a general analytical method has been introduced for rigorous calculation of the scattering harmonics for an arbitrary spacetime modulated impedance layer. To solve this problem, we combine the analytical method in [13] with MATLAB optimization. The Fourier coefficients of $C(z, t)$ are optimized until the beams from all input ports are fully concentrated at Port $N+1$.

\section{RESULTS}

Taking an example of a three-port power combiner, we assume that waves incident from Port 1 are directed at $\theta_{\mathrm{i}, 1}=45^{\circ}$, the spatial period is $D=58.1 \mathrm{~mm}$, and the modulation frequency $\omega_{\mathrm{M}}=0.062 \omega_{0}$, where $\omega_{0}=$ 
$2 \pi \times 10 \mathrm{GHz}$. Based on these parameters, the angles of Port 2 and Port 3 are calculated as $\theta_{\mathrm{i}, 2}=13.6^{\circ}$ and $\theta_{\mathrm{i}, 3}=-13.6^{\circ}$ from Eq. (2). Then we optimize the modulation function until the reflection coefficient to Port 4 is unity for all the incident beams. One set of the optimal material parameters is given in the caption of Fig. 1(b). As soon as the modulation function is optimized, the scattering harmonics for each incidence can be calculated from the mode-matching method [13], as shown in Fig. 1(b). One can see that, for a wave incident from Port 1, the reflected wave towards Port 4 (specular harmonic $n=0$ ) has amplitude close to the incident one $E_{0}$. Waves incident from Ports 2 and 3 are deflected towards Port 4 (as harmonics $n=1$ and $n=2$, respectively) with amplitudes close to $1.2 E_{0}$. All other propagating channels receive near-zero power. For the considered case, the receiving antenna must have minimum bandwidth of $\Delta \omega=2 \omega_{\mathrm{m}}=2 \pi \times 1.24 \mathrm{GHz}$ in order to incoherently collect the incident power from all the 3 ports.

\section{Conclusions}

Here, we have presented the concept of a multi-beam power combiner based on space-time modulated metasurfaces. By periodically modulating the surface properties of metasurfaces in both space and time, it is possible to realize perfect power concentration for plane waves from multiple directions to a single direction, without restrictions on incident phases and amplitudes. The method is rather general and can be applied in a large domain of electromagnetic waves, varying from the microwave to the optical domain.

\section{REFERENCES}

[1] D. M. Pozar, Microwave Engineering. John Wiley \& Sons, 2009, ch. 7.

[2] S. He and K. Liu, "On the possibility of a perfect power combiner," Progress In Electromagnetics Research, vol. 158, pp. 1-6, 2017.

[3] F. Smits, "Power combiners for incoherent waves," Tech. Rep., 1993.

[4] P. O. Minott and J. B. Abshire, "Grating rhomb diode laser power combiner," in Optical technologies for space communication systems, vol. 756. International Society for Optics and Photonics, 1987, pp. 38-49.

[5] M. K. Thumm and W. Kasparek, "Passive high-power microwave components," IEEE transactions on plasma science, vol. 30, no. 3, pp. 755-786, 2002.

[6] X. Wang, G. Ptitcyn, V. Asadchy, A. Díaz-Rubio, M. S. Mirmoosa, S. Fan, and S. A. Tretyakov, "Nonreciprocity in bianisotropic systems with uniform time modulation," Physical Review Letters, vol. 125, no. 26, p. 266102, 2020.

[7] A. Shaltout, A. Kildishev, and V. Shalaev, "Time-varying metasurfaces and lorentz non-reciprocity," Optical Materials Express, vol. 5, no. 11, pp. 2459-2467, 2015.

[8] H. Li and A. Alù, "Temporal switching to extend the bandwidth of thin absorbers," Optica, vol. 8, no. 1, pp. 24-29, 2021.

[9] S. Taravati and A. A. Kishk, "Space-time modulation: Principles and applications," IEEE Microwave Magazine, vol. 21, no. 4, pp. 30-56, 2020.

[10] M. Mirmoosa, G. Ptitcyn, V. Asadchy, and S. Tretyakov, "Time-varying reactive elements for extreme accumulation of electromagnetic energy," Physical Review Applied, vol. 11, no. 1, p. 014024, 2019.

[11] M. Liu, D. A. Powell, Y. Zarate, and I. V. Shadrivov, "Huygens' metadevices for parametric waves," Physical Review X, vol. 8, no. 3, p. 031077, 2018.

[12] D. L. Sounas and A. Alù, "Non-reciprocal photonics based on time modulation," Nature Photonics, vol. 11, no. 12, pp. 774-783, 2017.

[13] X. Wang, A. Díaz-Rubio, H. Li, S. A. Tretyakov, and A. Alù, "Theory and design of multifunctional space-time metasurfaces," Physical Review Applied, vol. 13, no. 4, p. 044040, 2020. 